\title{
Unsteady Adjoint Method for Aeroacoustic Propeller Optimization
}

\author{
Bambang I. Soemarwoto ${ }^{1}$, Harmen van der Ven ${ }^{1}$, Johan C. Kok ${ }^{1}$ and \\ Stevie R. Janssen ${ }^{2}$ \\ Royal Netherlands Aerospace Center NLR, 1059 CM Amsterdam, The Netherlands
}

The development of an adjoint method for design optimization of a propeller in an unsteady flow field is presented. The methodology follows the continuous-discrete approach, where the adjoint equations are formulated in a continuous form and afterwards discretized in the same way as the flow equations are discretized. The time-dependent flow and adjoint equations are solved respectively to determine the aeroacoustics performance and gradients of the functionals. The aerodynamic characteristics are obtained using NLR's CFD solver ENSOLV, while the acoustic characteristics are calculated using the Ffowcs Williams and Hawkings equation. Two aspects are highlighted, namely the development and verification of (i) the unsteady adjoint solver and (ii) the adjoint phase-lagged boundary condition. Computational results are presented for test cases ranging from an academic single airfoil to an industrially relevant propeller configuration.

\section{Nomenclature}

$\begin{array}{ll}A & =\text { Jacobian of the fluxes } \\ C_{d} & =\text { drag coefficient } \\ \boldsymbol{F} & =\text { fluxes } \\ \boldsymbol{I}, \boldsymbol{J} & =\text { generic functionals } \\ \boldsymbol{Q} & =\text { flow variables } \\ S & =\text { surface to be designed } \\ p & =\text { pressure } \\ p_{a} & =\text { acoustic pressure } \\ Y & =\text { Jacobian of the primitive flow variables } \\ t & =\text { time } \\ \vec{u} & =\text { velocity vector } \\ \boldsymbol{x} & =\text { coordinates of } S \\ \boldsymbol{\theta} & =\text { vector of design variables } \\ \tau_{w} & =\text { wall shear stress } \\ \lambda & =\text { adjoint variables } \\ \Omega & =\text { flow domain }\end{array}$

\section{Introduction}

Due to demand for reduced fuel burned and emissions of carbon and NOx, there is a growing interest in propeller propulsion that covers a wide range of applications. In addition to applications for conventional turboprop configurations, novel configurations are emerging such as new concepts of high-speed helicopters, electrical aircraft with distributed propulsion, air taxis, and commercial drones. At the same time, societal concerns demand a reduction in perceived noise. For a sustainable economic growth, the aviation industry faces a tremendous challenge to meet the combined demands, where the aeroacoustic performance of the propeller must be improved. The propeller must be

\footnotetext{
${ }^{1}$ Senior Scientist, Flight Physics and Loads Department.

2 Medior Scientist, Flight Physics and Loads Department.
} 
optimized to be aerodynamically more efficient and at the same time acoustically more silent. In terms of the FlightPath 2050 goals set by Advisory Council for Aeronautical Research in Europe (ACARE) [1], the design improvement of the aerodynamics and acoustics must contribute to the reduction of CO2, NOx and perceived noise emissions by $75 \%, 90 \%$ and $65 \%$, respectively.

Apart from the multidisciplinary character of the challenge, i.e. aerodynamics and acoustics, the physics of the problem is genuinely unsteady. A good example is a recent study [2] for a compound helicopter configuration, Airbus RACER. In hover conditions, the wingtip-mounted propellers in a pusher configuration must perform under a downwash of the overhead rotor. During cruise the propellers must perform under an influence from the box wing and wing tip. The propeller design faces a challenge of a wide variation in thrust and blade pitch that must be realized under stringent power constraints. Another example is the NASA X-57 having a combination of propellers mounted on the wing tip and twelve smaller propellers distributed along the wing span [3]. The wingtip-mounted propellers are operated throughout the flight envelope, while the distributed propellers are operated only in low-speed region in takeoff and landing segments. Both types of the propellers experience a significant interactional flow due to the presence of the wing and the other propellers.

In order to sufficiently take the flow unsteadiness into account, the capability and affordability of deploying a high-fidelity time-accurate unsteady modeling throughout the design optimization procedure should be pursued. The alternative approach of deploying a low-cost low-fidelity modeling brings risk of missing the physics that may be important for the design, e.g. as indicated in Ref. [4]. It is well known that an efficient way of design optimization with a high-fidelity model such as Computational Fluid Dynamics (CFD) is to apply the adjoint method. In this case, the adjoint method must be capable of dealing with an unsteady flow involving the rotating blades. The interest to have such a capability is reflected in recent works presented in Refs. [5-7].

Here, the development of an adjoint methodology is presented for optimizing propellers in a periodically unsteady flow field. A systematic step-by-step approach is followed, using test cases of increasing complexity, starting from an academic single airfoil test case to an industrially representative propeller configuration. Each step allows a verification of the sensitivities and gradients with respect to the surface variation and design variables, respectively, which in turn serves as a basis for the subsequent verifications.

First, a general formulation of the optimization problem is written in Section III, illustrating the generality of the method. Section IV describes the basis for the determination of the aerodynamic and acoustic performance metrics. A summary of the adjoint problem is presented in Section V. This covers the development of an unsteady adjoint solver and the application of a phase-lagged adjoint boundary condition. The solution procedure and means of verification are explained in Section VI, and its relevance for propeller optimization is presented in Section VII. Finally, conclusions are drawn in Section VIII.

\section{Optimization problem}

The design optimization problem is formulated as a constrained multi-objective problem that can be expressed in a generic form as

$$
\text { minimize } \quad \boldsymbol{J}=\boldsymbol{J}(\boldsymbol{Q}, \boldsymbol{\theta})=\iint \psi(\boldsymbol{Q}(\boldsymbol{x}, t), \boldsymbol{x}) d S d t, \quad \boldsymbol{x} \in S(\boldsymbol{\theta}, t), \quad t_{0} \leq t \leq t_{1}
$$

subject to equality constraints:

$$
\boldsymbol{G}_{f}(\boldsymbol{Q}, \boldsymbol{\theta})=0 \text { and } \boldsymbol{G}_{g}(\boldsymbol{\theta})=0
$$

and non-equality constraints:

$$
\boldsymbol{H}_{f}(\boldsymbol{Q}, \boldsymbol{\theta}) \leq 0 \text { and } \boldsymbol{H}_{g}(\boldsymbol{\theta}) \leq 0
$$

where $\boldsymbol{J}, \boldsymbol{G}$, and $\boldsymbol{H}$ denote the vectors of objectives, equality constraints and inequality constraints, respectively. The subscripts $f$ and $g$ refer to aerodynamic/aeroacoustic and geometric constraints, respectively. The time span $t_{0} \leq t \leq$ $t_{1}$ is equal to the period of unsteadiness associated with the blade-passing frequency. The design variables are denoted by an $n_{\theta}$-dimensional vector $\boldsymbol{\theta}$ defining the coordinates $\boldsymbol{x} \in S(\boldsymbol{\theta}, t)$ of the shape to be optimized. The equality constraints $\boldsymbol{G}_{f}$ always include the flow equations. Furthermore, $\boldsymbol{J}, \boldsymbol{G}_{f}$ and $\boldsymbol{H}_{f}$ will include aerodynamic and/or 
aeroacoustic functionals, generally denoted as $I_{a e}$ and $I_{a c}$, which are defined in the following sections. The vector $\boldsymbol{Q}=(\rho, \vec{u}, p)$ denotes the state (flow) variables, with $\rho, \vec{u}$ and $p$ the density, velocity vector and pressure, respectively.

\section{Flow and acoustic analysis}

The aerodynamic characteristics are determined using NLR's CFD solver ENSOLV [8] that solves the timedependent Reynolds-averaged Navier-Stokes (RANS) equations,

$$
\frac{\partial \boldsymbol{Q}}{\partial t}+\vec{\nabla} \cdot \boldsymbol{F}(\boldsymbol{Q})=0 \quad \text { in } \Omega,
$$

with $\Omega$ the flow domain and $\boldsymbol{F}$ the fluxes. The flow equations are subject to the boundary conditions,

$$
B(\boldsymbol{Q}, \boldsymbol{x}, t)=0 \quad \text { on } S(\boldsymbol{\theta}, t),
$$

consisting of the no-slip and adiabatic wall boundary conditions. A variant of the two-equation $k-\omega$ turbulence model is added to close the system of equations. The aerodynamic functionals to be considered are force and moment coefficients defined as a surface integral of pressure and/or wall shear stress that can be written in a general form as:

$$
I_{a e}=\iint \psi\left(p, \tau_{w}\right) d S d t
$$

where $p$ and $\tau_{w}$ are the pressure and wall shear stress.

The Ffowcs Williams and Hawkings (FWH) analogy [9] is used to determine the acoustic characteristics. The farfield pressure is decomposed into thickness, loading and quadrupole contributions to the noise, where the quadrupole contribution is neglected. The thickness contribution is due to the motion of the geometry only, while the loading contribution also depends on the pressure on the geometry. The so-called advanced time formulation [10] is applied due the advantages that it scales linearly with the number of source time steps, and it is independent of the number of observer time steps. The acoustic functional is expressed in a general form as

$$
I_{a c}=\sum_{i=1}^{N_{o b s}} \int\left(p_{a}\left(p, x_{i}, t\right)-p_{\infty}\right)^{2} d t
$$

comprising the thickness and loading noise. Here $N_{o b s}$ is the number of observer locations $x_{i}$, and $p_{a}\left(p, x_{i}, t\right)$ is the acoustic pressure at the observer's location and time. The acoustic pressure is computed as the sum of the loading and thickness noise from the FWH formulation.

\section{Adjoint problem}

A Lagrangian is defined which augments the functional (1) with the state constraints (4) and (5) by means of the adjoint variables. Through a variational analysis of the Lagrangian, the time-dependent adjoint equation can be derived in the following form:

$$
-\frac{\partial \lambda}{\partial t}-\mathrm{A}^{\top} \cdot \nabla \lambda-\mathrm{Y}^{\top} K=0 \quad \text { in } \Omega
$$

where $\lambda=\left(\lambda_{1}, \vec{\lambda}, \lambda_{5}\right)^{\top}$ denotes the vector of adjoint variables, where $\vec{\lambda}=\left(\lambda_{2}, \lambda_{3}, \lambda_{4}\right)^{\top}$ may be considered as the socalled adjoint velocity. A is the Jacobian of the fluxes with respect to the conservative flow variables. The second and third terms on the left-hand side of the equation are the adjoint convective and viscous terms, where $K$ contains second derivatives of $\lambda$ and $Y$ is the Jacobian of the mapping from primitive to conservative flow variables. The minus sign of the time derivative is maintained here to indicate that the time integration is done in the opposite direction to the primal (flow) problem. It should be noted that in the current implementation the eddy viscosity is frozen, leaving out the terms originating from the turbulence model equations. The derivation of the adjoint boundary conditions leads to an expression having a similar form as the flow boundary conditions (5).

For the aerodynamic functional $I=I_{a e}$ as objective $J$, the adjoint boundary conditions at all time $t$ can be summarized as: 


$$
\vec{\lambda} \cdot \vec{n}=\frac{\partial \psi}{\partial p}-(\vec{u} \cdot \vec{n}) \lambda_{5}, \quad \vec{\lambda} \cdot \vec{s}=-\frac{\partial \psi}{\partial \tau_{w s}}-(\vec{u} \cdot \vec{s}) \lambda_{5}, \quad \vec{\lambda} \cdot \vec{t}=-\frac{\partial \psi}{\partial \tau_{w t}}-(\vec{u} \cdot \vec{t}) \lambda_{5}, \quad \vec{\nabla} \lambda_{5} \cdot \vec{n}=0
$$

where $\vec{n}$ is the unit normal vector on the surface, while $\vec{s}$ and $\vec{t}$ are the unit tangential vectors.

For the acoustic functional $I=I_{a c}$ as objective $J$, a simple form of an integral over the surface and time cannot be written. As a consequence, the boundary conditions of the adjoint equation have been obtained in a discrete way. The derivative of the functional with respect to pressure is then evaluated as

$$
\frac{\partial I}{\partial p} \approx \frac{\partial I_{h}}{\partial p_{i}^{n}} \frac{\partial p_{i}^{n}}{\partial p}
$$

where the subscript $h$ denotes discretization, and $p_{i}^{n}$ is the pressure on a discrete face $i$ at time $t=t^{n}$. This pressure is assumed to be the face-averaged pressure on the discrete space-time face $S_{i}^{n}=S_{i} \times\left[t_{0}, t_{1}\right]$,

$$
p_{i}^{n}=\frac{1}{\left|S_{i}^{n}\right|} \int_{S_{i}^{n}} p(x, t) d x d t
$$

The derivative is then calculated by means of an automatic differentiation code Tapenade [11] for a given pressure distribution and geometry of the surface. In brief, the resulting adjoint boundary conditions for the discretized acoustic functional can be expressed as

$$
\vec{\lambda} \cdot \vec{n}=\frac{1}{\left|S_{i}^{n}\right|} \frac{\partial I_{h}}{\partial p_{i}^{n}}-(\vec{u} \cdot \vec{n}) \lambda_{5}, \quad \vec{\lambda} \cdot \vec{s}=-(\vec{u} \cdot \vec{s}) \lambda_{5}, \quad \vec{\lambda} \cdot \vec{t}=-(\vec{u} \cdot \vec{t}) \lambda_{5}, \quad \vec{\nabla} \lambda_{5} \cdot \vec{n}=0
$$

\section{Method Development and Means of Verification}

\section{A. Unsteady adjoint solver}

The starting point of the development is the existing steady adjoint solver ENSENS available at NLR, which has been used in various projects (e.g. Refs. [12,13]). ENSENS is a three-dimensional extension of a two-dimensional adjoint methodology presented in Refs. [14,15]. It is based on a continuous formulation of the adjoint equations, which are discretized in a similar way as the flow equations. This approach allows a re-use of much of the solution algorithm and code structure of the flow solver ENSOLV that solves the time-dependent RANS equations. In developing the unsteady adjoint solver, the same continuous-discrete approach is followed such that the time-accurate solution procedure with the dual-time stepping and multigrid scheme of the flow solver can be fully adopted.

To facilitate an efficient development and verification, a simple single-element airfoil is considered first. An ultralow Reynolds number allowing a fully laminar flow around a single Eppler 387 airfoil rotating in a still air is defined (see Figure 1). The rotating airfoil exerts a drag force and produces an acoustic signal for an observer located on the plane of rotation.

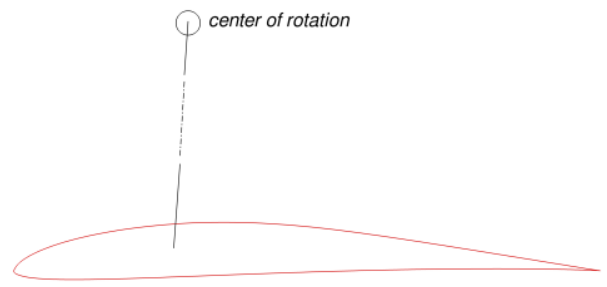

Figure 1 Airfoil rotating in still air.

Two situations can now be considered. In the first situation, the flow equations are formulated in a frame of reference that follows the rotation of the airfoil. In this rotating frame, the flow field is steady. Accordingly, both the flow and adjoint equations can be solved using the existing steady solution procedure. This provides a reference steady 
adjoint solution and its corresponding grid-point geometry sensitivity. This reference sensitivity has been verified by means of a comparison of the gradients shown in Figure 2 according to the parameterization scheme described in Ref. [14]. With this verified geometry sensitivity, it can be deduced that the reference steady adjoint field is correct.

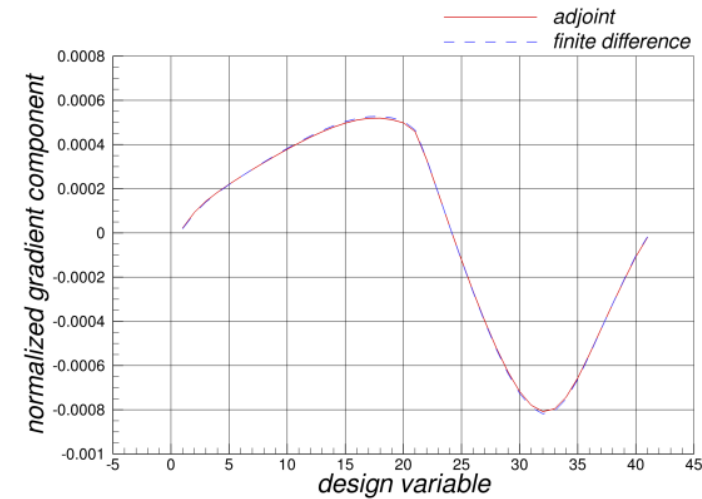

Gradient of drag coefficient $C_{d}$

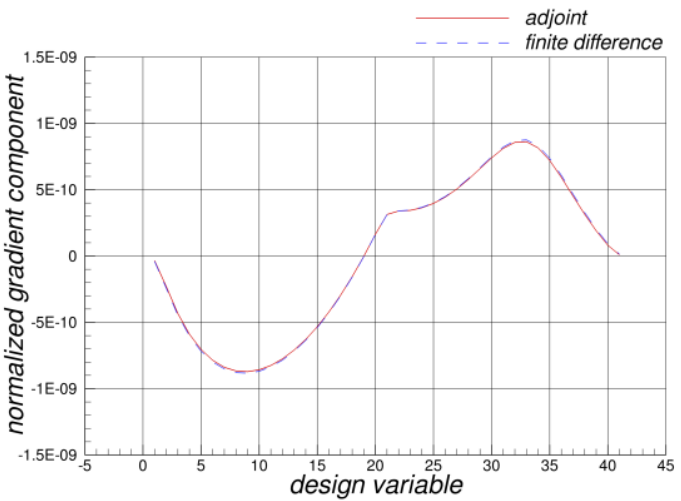

Gradient of noise $I_{a e}$

Figure 2 Comparison between finite-difference and adjoint gradients.

In the second situation, the flow equations are formulated in the inertial frame of reference, involving a rigid motion of the grid following the rotation of the airfoil. In the unsteady solution procedure the flow equations are integrated forward in time from $t_{0}$ to $t_{1}$, providing an instantaneous flow solution at each time step associated with the current position of the airfoil. These instantaneous solutions are stored as input for the subsequent adjoint solution procedure.

After a periodic solution (which is also steady in this case) of the pressure field has been obtained, the adjoint equations are integrated using a similar solution procedure. Starting from the final position of the airfoil, with a zero adjoint field as an initial condition, the adjoint equations are integrated backward in time until the periodic adjoint solution is obtained (also steady in this case). This resulting adjoint solution is to be compared with the reference steady adjoint solution obtained in the first situation. In the adjoint solution procedure, the airfoil is moving backward from the final towards the initial position as depicted in Figure 3.

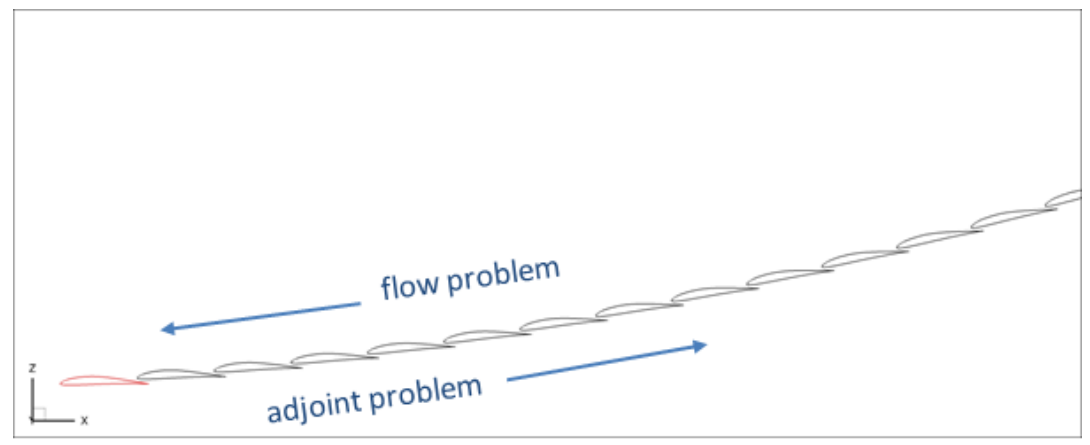

Figure 3 Airfoil moving forward (backward) in the flow (adjoint) problem.

Snapshots of the (transient) adjoint solution at $t=t_{1}-\omega\left(t_{1}-t_{0}\right)$ are shown in Figure 4 for the drag functional. At the end of the solution procedure, the steady-state reference adjoint solution and geometry sensitivity have been fully recovered by the unsteady adjoint solution procedure, as shown in Figure 5 and Figure 6. 


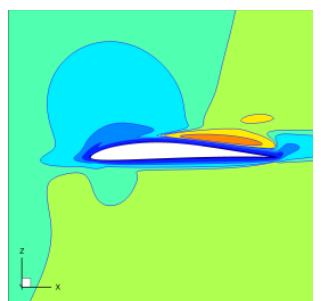

$\omega=1 / 360$

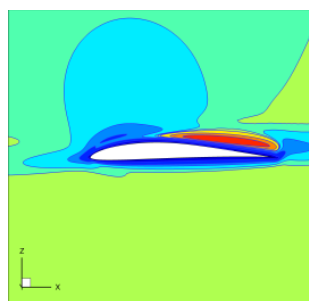

$\omega=2 / 360$

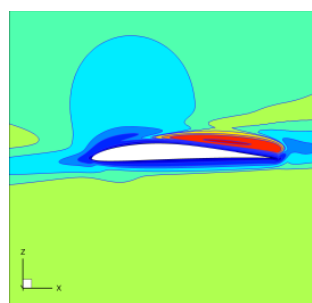

$\omega=4 / 360$

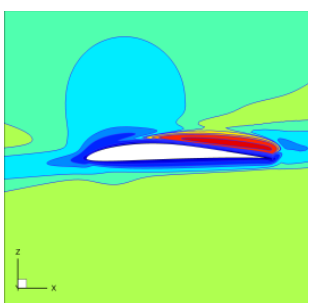

$\omega=8 / 360$

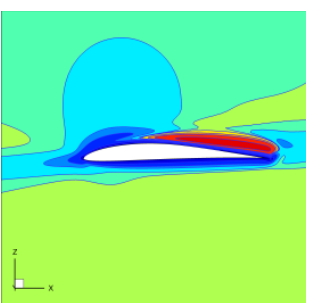

$\omega=16 / 360$

Figure 4 Instantaneous adjoint pressure contours.

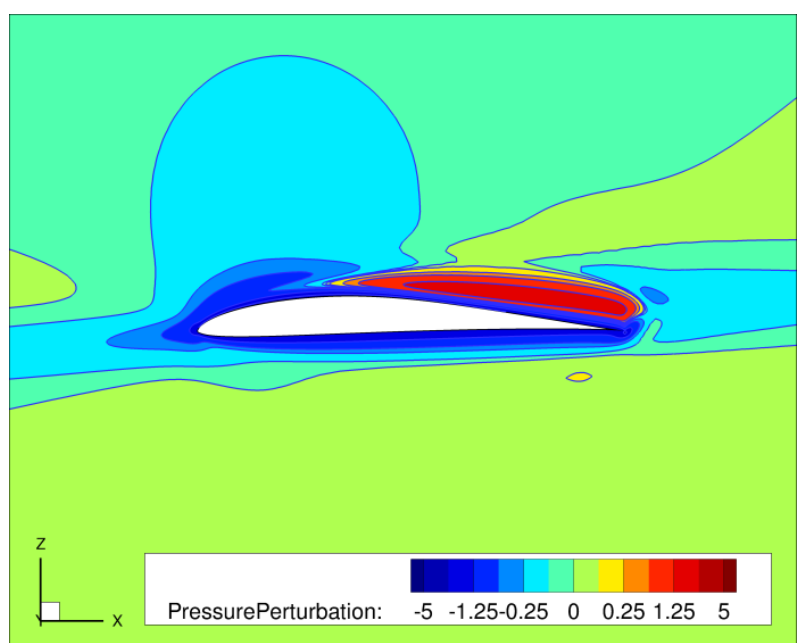

(a) steady adjoint pressure contours

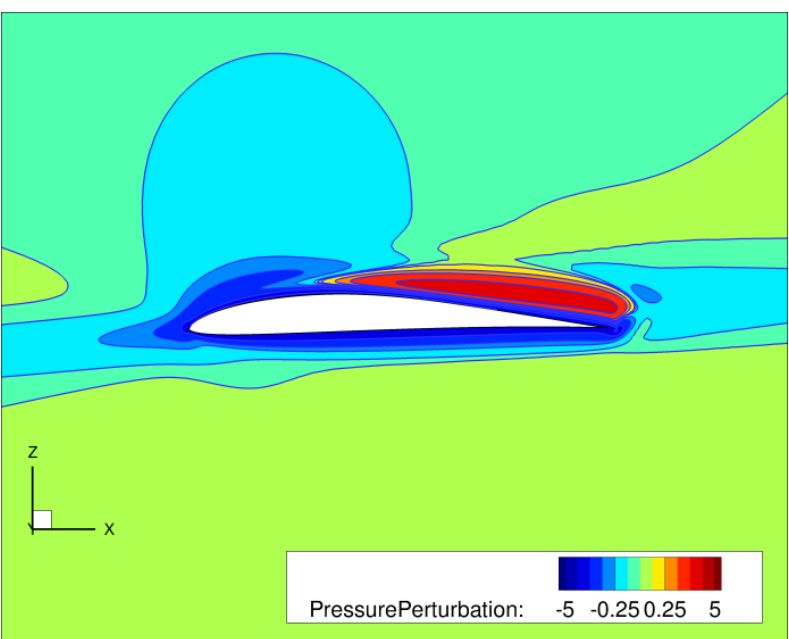

(b) unsteady adjoint pressure contours $(\omega=1)$

Figure 5 Comparison of the adjoint solutions obtained by the steady and unsteady solver for $C_{d}$.

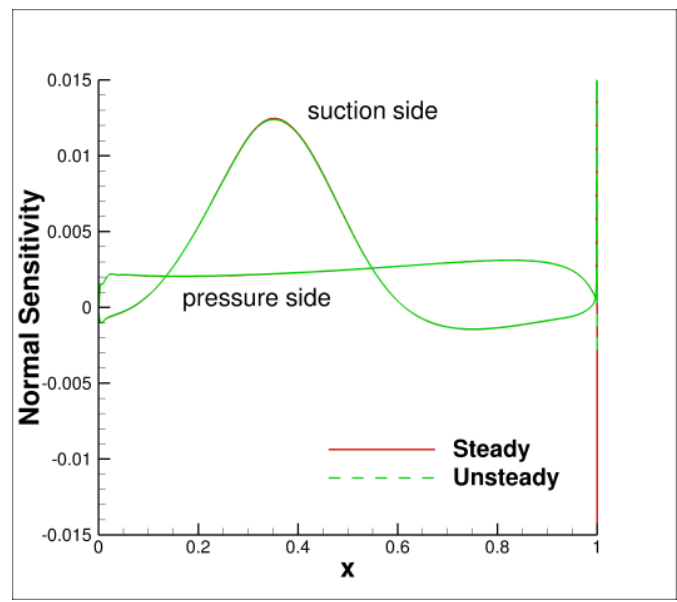

Figure 6 Comparison of the geometry sensitivities obtained by the steady and unsteady solver for $C_{d}$.

In a similar way, the verification is done for the noise functional $I_{a e}$, resulting in a close agreement shown in Figure 7 and Figure 8. It should be noted here that the pressure signals received by the observer are fundamentally unsteady phenomena. Accordingly, the steady adjoint solution (sensitivity) obtained in the rotating frame of reference should be compared with a summation over one period of the discrete instantaneous unsteady adjoint solution (sensitivity) in the inertial frame of reference.

It can be deduced that, through a chain-rule differentiation, the close agreement on the level of surface sensitivity shown in Figure 6 and Figure 8 will lead to a close agreement on the level of gradient consistent with Figure 2. 


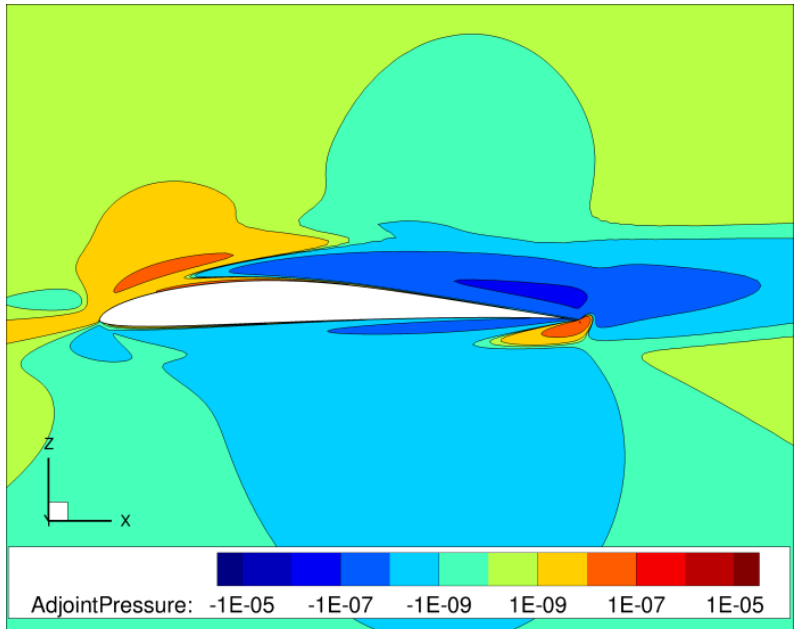

(a) steady adjoint pressure contours

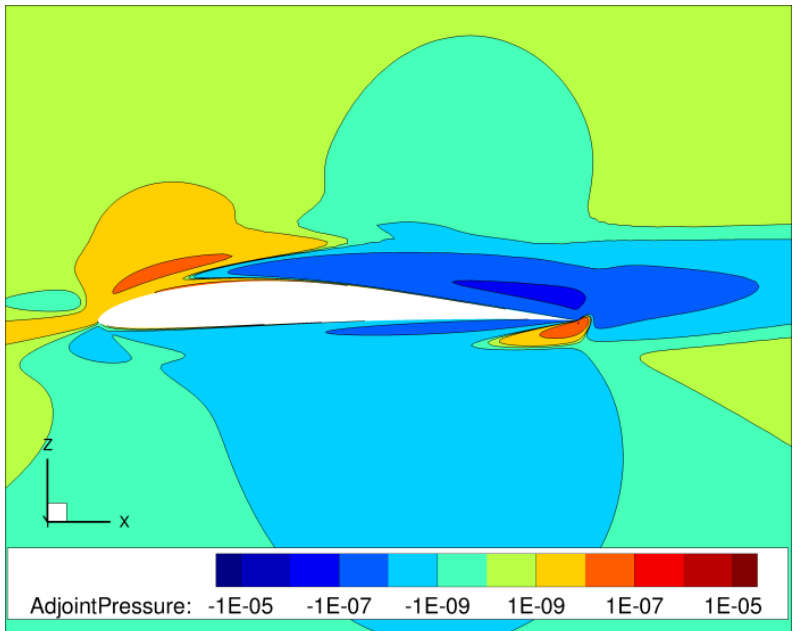

(b) unsteady adjoint pressure contours summed over one period

Figure 7 Comparison of the adjoint solutions obtained by the steady and unsteady solver for noise.

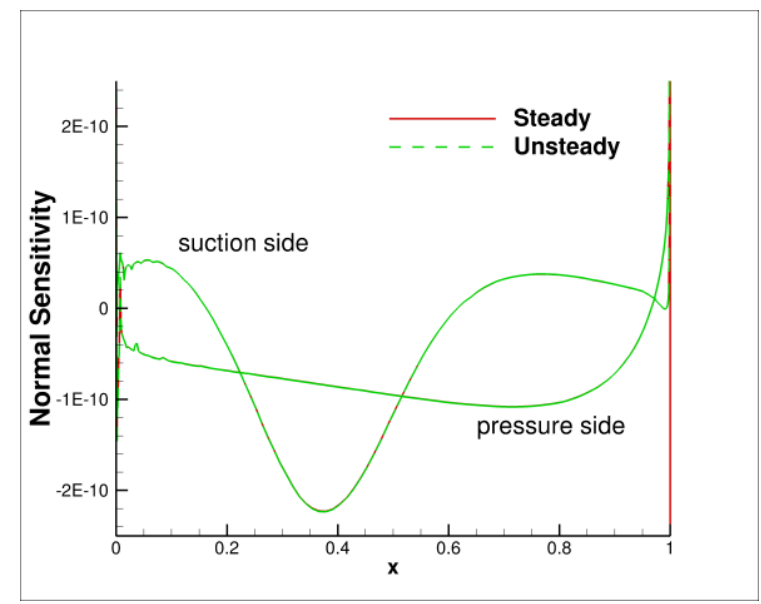

Figure 8 Comparison of the sensitivities obtained by steady and unsteady solver for noise.

\section{B. Phase-lagged boundary condition}

In a real-life application, the process of writing and reading the transient flow solution at each time step, respectively by the flow solver and adjoint solver, may amount to heavy disk I/O loads. This can be a challenge in case of a high number of blades, where up to 8 blades for a propeller and around 20 blades for a typical engine fan can be expected.

In total for a fine grid and a sufficient temporal resolution, the data volume that needs to be written and read can reach an order of magnitude of Tbytes for one optimization iteration and one functional only. The implication of such a large I/O load to the turn-around time of a design optimization cycle is difficult to quantify precisely. However, within a variation of the $\mathrm{I} / \mathrm{O}$ bandwidth on a typical high performance cluster computing facility, the portion of the $\mathrm{I} / \mathrm{O}$ operations alone spent in one design cycle may be prohibitive, significantly exceeding the computing (execution) time [16].

A phase-lagged (a.k.a. chorochronic) scheme $[17,18]$ is considered to mitigate the heavy I/O loads and reduce the wall clock time of a design cycle. In such a scheme, only a single blade passage is computed, using appropriate boundary conditions that take the phase lag between the 'periodic' side boundaries into account. This means that data 
of only one single blade passage needs to be stored at each time step. As a result, the reduction of the I/O loads can be expected to be proportional to the number of blades.

Again, in order to facilitate an efficient development and verification, a simple test case is defined. A comparison can then be made between results obtained using a full configuration and those obtained using only one single blade passage with the phase-lagged boundary condition.

The NASA Rotor-67 geometry [19] having 22 blades is simplified. Figure 9a shows that a cut of a unit grid cell was made across the mid-span of the rotor to construct a 2.5D-configuration. An inflow boundary condition is applied where a periodic distribution of the axial momentum is specified to simulate a situation around the propeller for an inclined incoming flow. The resulting instantaneous flow solution is presented in Figure $9 \mathrm{~b}$.

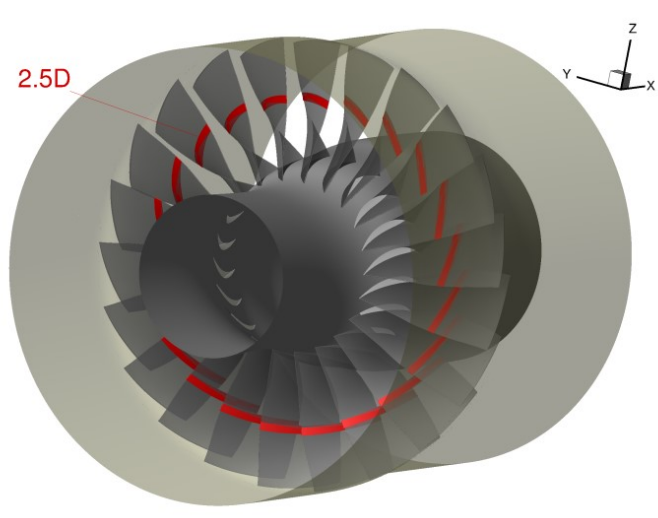

(a)

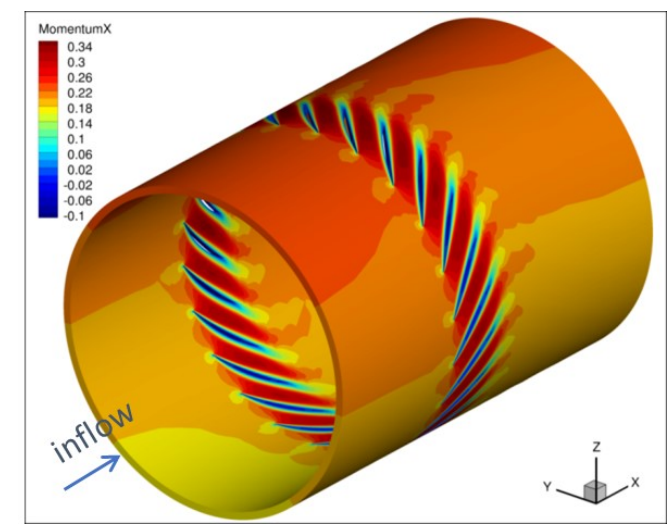

(b)

Figure 9 2.5D geometry and flow solution with a periodic inflow boundary condition.

Now, the full-rotor configuration is reduced to a single blade passage configuration. A characteristic phase-lagged boundary condition is applied as depicted in Figure 10, effectively coupling the flow variables of both sides of the passage.

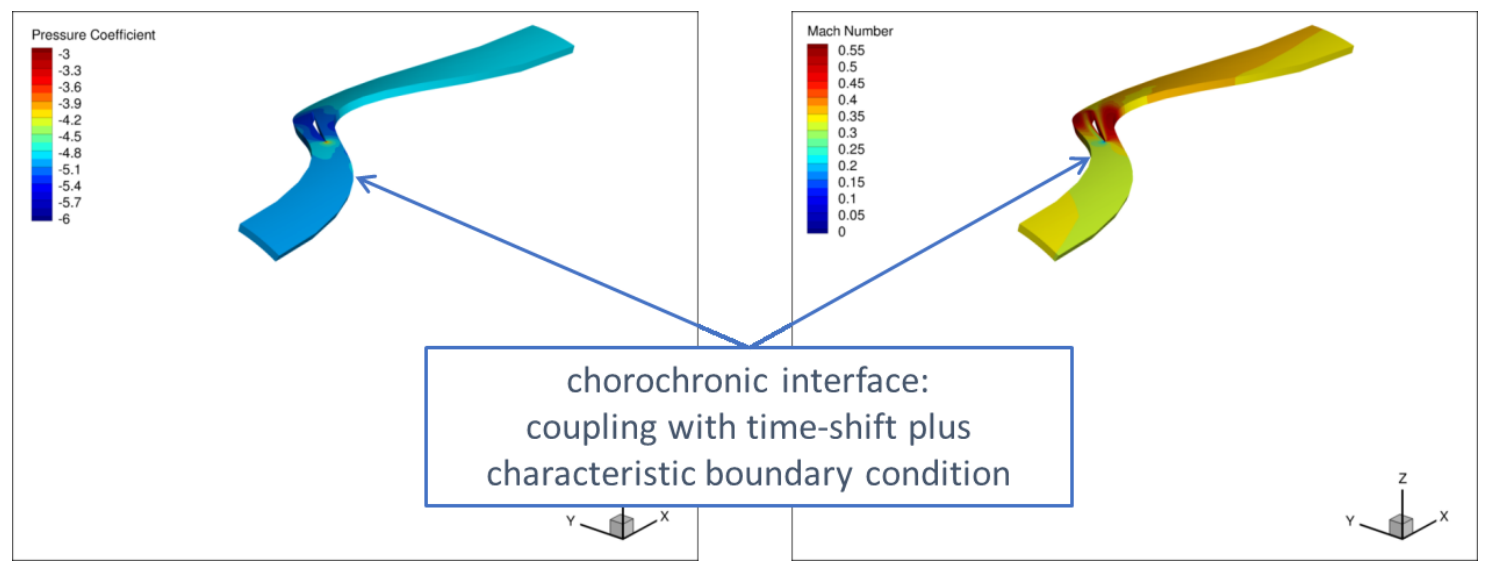

Figure 10 single-blade phase-lagged (or chorochronic) configuration.

Figure 11 shows a comparison between the flow and adjoint solutions obtained using the full-rotor configuration and those obtained using the single-blade phase-lagged configuration. The flow solution is shown in terms of contours of the Mach number, while the adjoint solution is shown in terms of contours of the first component of the adjoint velocity $\vec{\lambda}$. If observed closely, there are only very minor differences that can possibly be attributed to the application of the characteristic coupling between the phase-lagged interfaces. It should be mentioned that the differences become smaller when the grid resolution is increased. A comparison of the grid-point geometry sensitivity in the normal direction to the surface is also shown in Figure 12, demonstrating a very close agreement. 

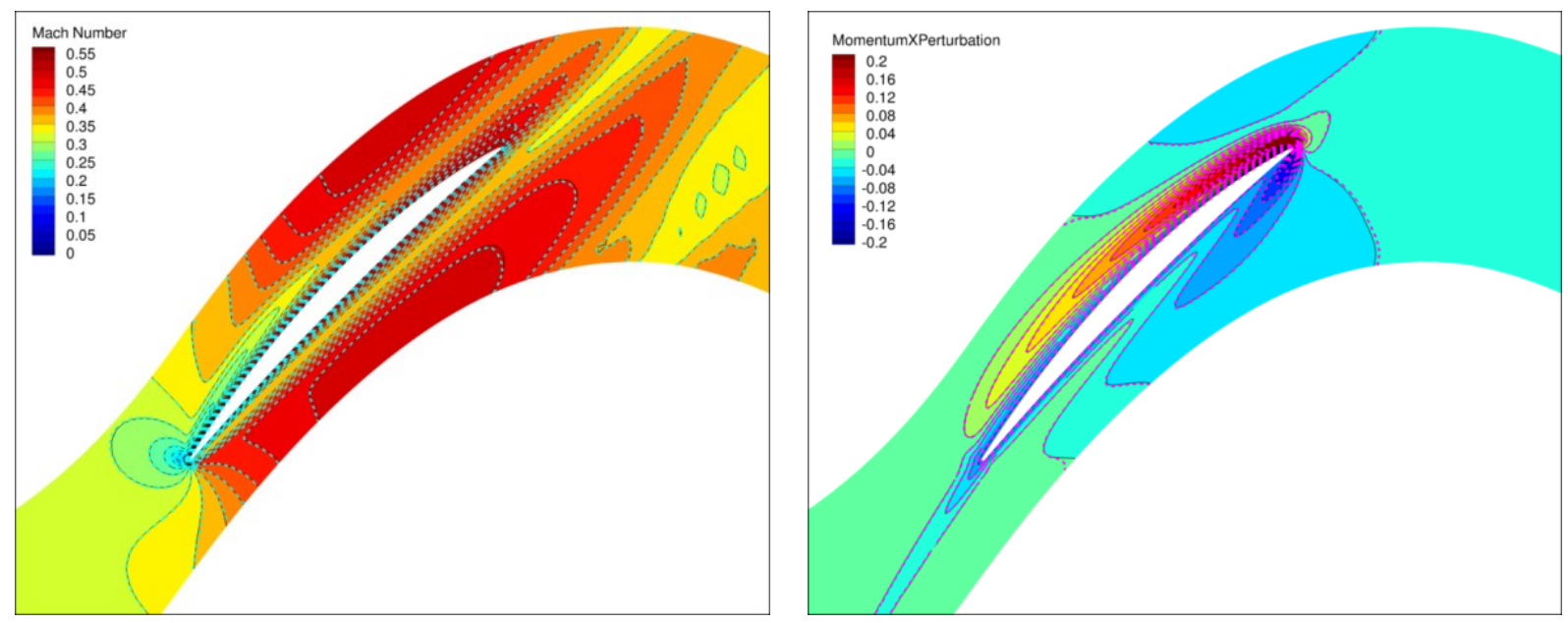

Figure 11 Comparison of the flow (left) and adjoint (right) solutions obtained using the full-rotor (flood) and single-blade phase-lagged (dashed lines) configuration.

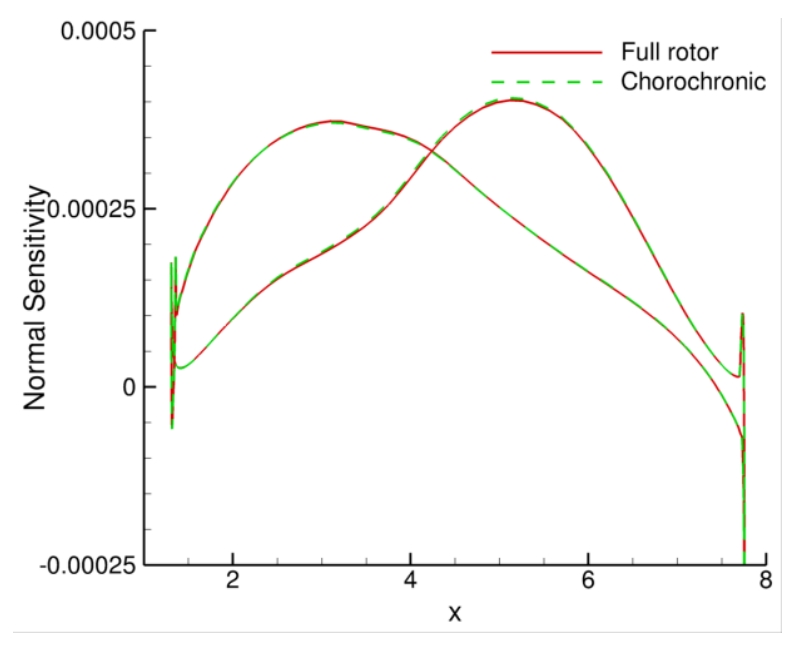

Figure 12 Comparison of the sensitivities.

\section{Application for Propeller Optimization}

The unsteady adjoint methodology described above can now be applied to an industrially relevant propeller configuration. ONERA HAD-1 three-bladed propeller is considered. This propeller was designed for an electrical or hybrid CTOL/VTOL (Conventional/Vertical Take-Off and Landing) concept. A typical result of a steady simulation of the propeller in an axial incoming flow is shown in Figure 13, for which a 1-BP (one blade passage) domain can be used. The polar curve presented in the figure is constructed based on five blade pitch angles. The design point is indicated by the unit value of the normalized thrust and torque, which is approximately given by the blade pitch of 48 degrees.

The adjoint analysis at the blade pitch of 45 degrees is conducted to obtain the gradient with respect to the design variables. In total there are 147 design variables equally distributed over 7 control stations along the blade span including the tip. The shape of the airfoil at each control station is determined by 21 design variables [13]. At an intermediate position between adjacent control stations, the airfoil shape is determined by means of blending the two mode shapes at the adjacent stations.

A close agreement between the finite-difference and adjoint gradients of the thrust, torque (representing power) and acoustic functionals is demonstrated in Figure 14. These three functionals are all that is needed for an aeroacoustic optimization in terms of propeller efficiency and noise. Larger discrepancies between the gradients can be observed 
regarding the noise functional. This can partly be attributed to the step size of the finite-difference perturbation that may not be equally suitable for both the RANS (CFD) and FWH analyses.

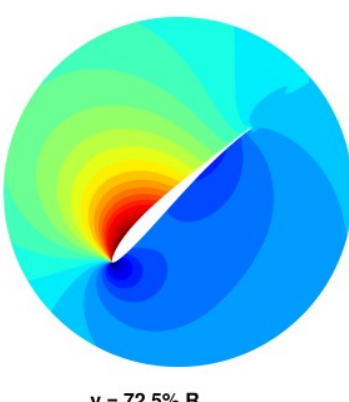

$y=72.5 \% R$
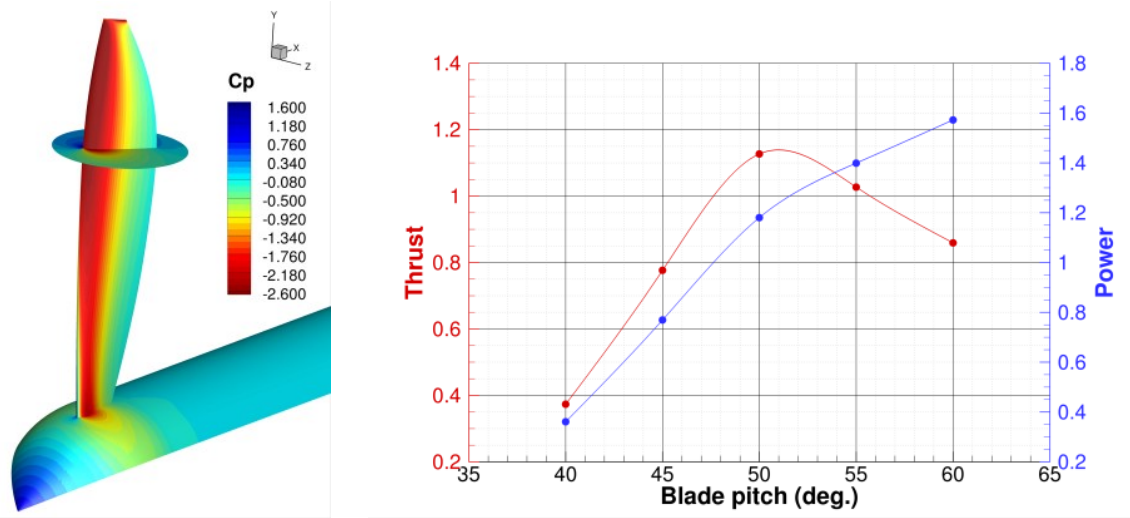

Figure 13 ONERA's HAD-1 propeller characteristics.

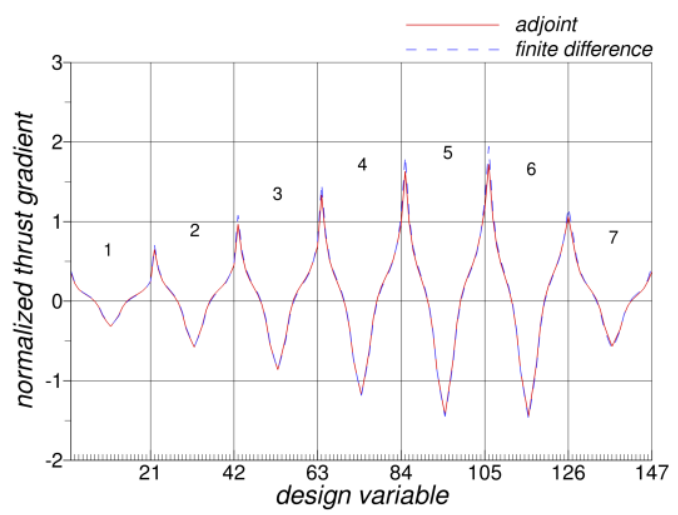

(a)

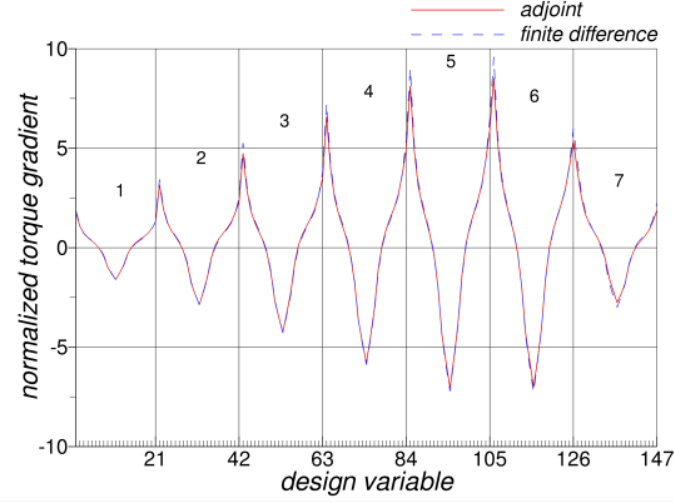

(b)

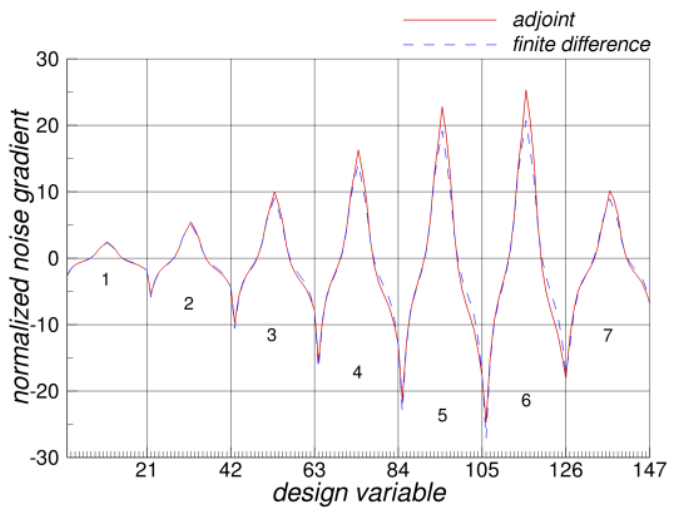

(c)

Figure 14 Verification of the gradients of the thrust, torque and acoustic functionals.

The next verification considers a 3-BP instead of 1-BP domain for the same axial incoming flow condition. The same steady-state solution procedure in the rotating frame of reference is still used. This verification is necessary to ensure a consistent chain-rule differentiation from local surface sensitivities on the three blades to the 147 design variables defining the reference blade, in order to produce three exactly the same blade geometries throughout the course of optimization. Figure 15 confirms consistency of the chain-rule differentiation. 


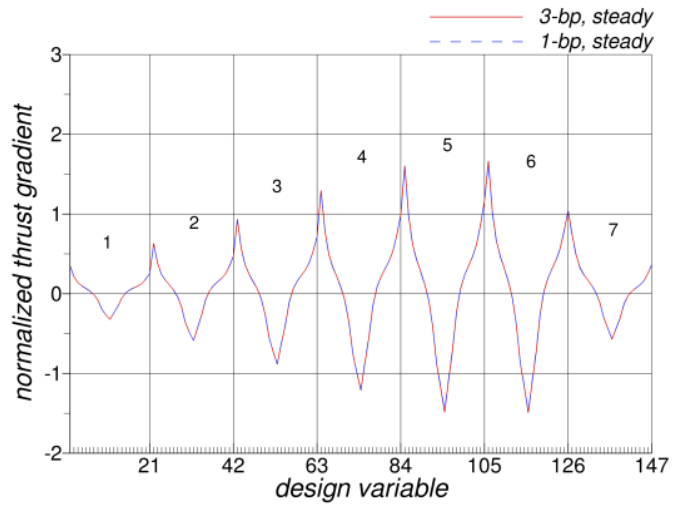

Figure 15 Verification of the thrust gradient of 3-BP domain procedure.

Now, another verification can be performed by rotating the propeller in the inertial frame. This requires an unsteady solution procedure for both the forward (flow) and adjoint problems. It should be noted that the term local (for the surface sensitivities) now refers to a specific spatial and temporal location, as the propeller rotates backward in time during the unsteady adjoint simulation. For this test case, the blade pitch angle is adjusted to 48 degrees in order to approach the design point indicated in Figure 13. The grid resolution has also been reduced to the medium level with 3 million grid cells, instead of 24 million grid cells on the fine grid level. The reason for this reduction is to limit the $\mathrm{I} / \mathrm{O}$ effort that is required to write and read the flow solutions at all time steps.

The unsteady flow simulation starts with one revolution on the coarse grid with 3 degrees per time step, and subsequently continues with three revolutions on the medium grid with 2 degrees per time step. The flow solutions are stored only during the last revolution. Figure 16a shows that the thrust has converged to a steady value. The adjoint simulation starts from the last time step backward in time, reversing the rotation of the propeller. Figure $16 \mathrm{~b}$ presents the time history of the sensitivity to geometry scaling in the $x$-direction, which shows that convergence has been achieved after two revolutions. Figure 16c confirms that both the steady (in the rotating frame) and unsteady (in the inertial frame) adjoint solution procedure give the same gradient of the thrust.

Introducing an angle of attack of 4 degrees, a genuinely unsteady problem is created, which can only be dealt with unsteady solution procedures. The same strategy is applied as above, i.e. the flow simulation starts with one revolution on the coarse grid followed by three revolutions on the medium grid. Figure 17a shows that the thrust has converged to a periodical solution. Similar as above, the adjoint simulation starts from the last time step backward in time, reversing the rotation of the propeller. After just two revolutions, the solution has become periodic as shown in Figure $17 \mathrm{~b}$. This solution is obtained using the conventional three-bladed configuration, requiring the flow solutions in the full 3-BP domain to be stored at all time steps during the last forward revolution.

Efficiency improvement can now be assessed if the phase-lagged boundary condition for the adjoint solution procedure is applied. First, this scheme requires the flow solutions of only 1-BP domain to be stored, directly delivering a significant performance gain in terms of three times less storage and I/O loads. The 1-BP adjoint computation delivers the sensitivities to the geometry of a single blade only. Therefore, the time histories of the 1-BP and 3-BP adjoint solutions have to be compared for a single blade as well (instead of all three blades as in Figure 17b). This is done in Figure 17c, which shows that the time history of the adjoint simulation with the phase-lagged boundary condition is practically identical to the full 3-BP computation and therefore converges at the same rate. Figure $17 \mathrm{~d}$ confirms that both the conventional and phase-lagged procedures give the same gradient. This also means a significant performance gain in terms of processing time. As the computational domain is three times smaller, the computing time is three times faster.

\section{Conclusions}

The development of an unsteady adjoint solver has been presented. A sequence of verification has been conducted, utilizing test cases of increasing complexity from a simple academic to an industrially relevant test case. The aim of the verification is two-fold, to show that (i) the unsteady adjoint solution procedure will lead to the correct sensitivity or gradient of the aerodynamic and acoustic functionals, and (ii) a significant performance gain proportional to the number blades can be achieved by means of a phase-lagged boundary condition. Based on the results shown for the thrust functional, it can be deduced that the achieved verification is also valid for the torque (power) and noise 
functionals. In conclusion, a sound foundation has been achieved to allow a correct and affordable aeroacoustic propeller design optimization that need to be performed in future work, involving unsteady interactional flows.

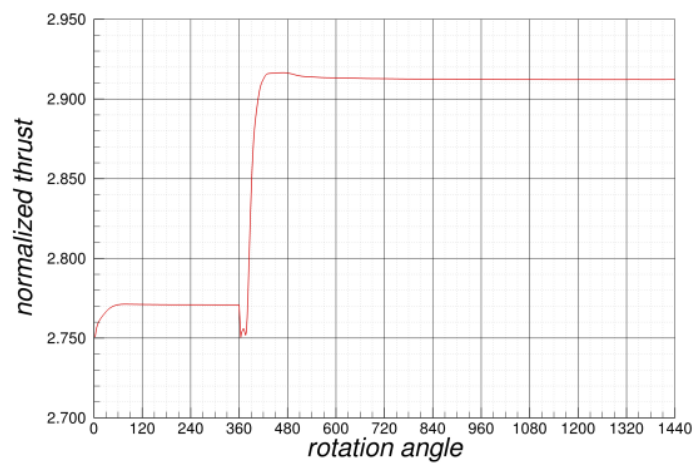

(a) Flow simulation (unsteady procedure)

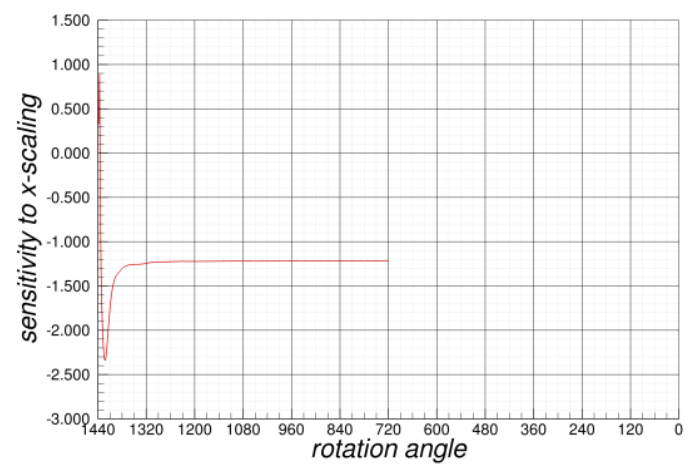

(b) Adjoint simulation (unsteady procedure)

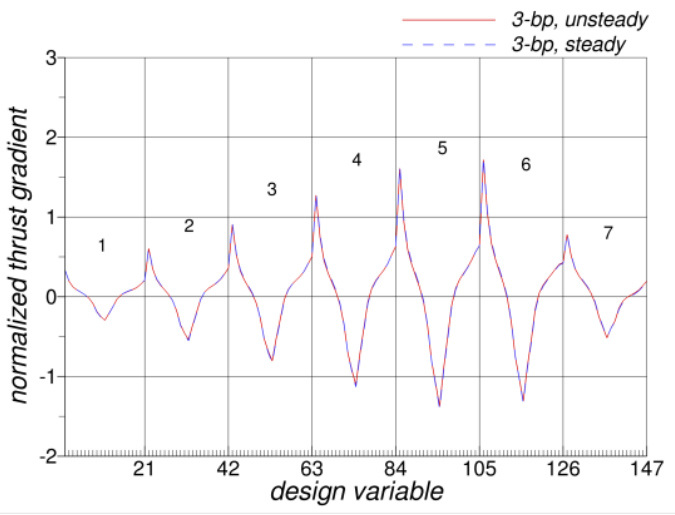

(c) Comparison of the gradients.

Figure 16 Verification of the thrust gradient obtained by the unsteady adjoint procedure. 


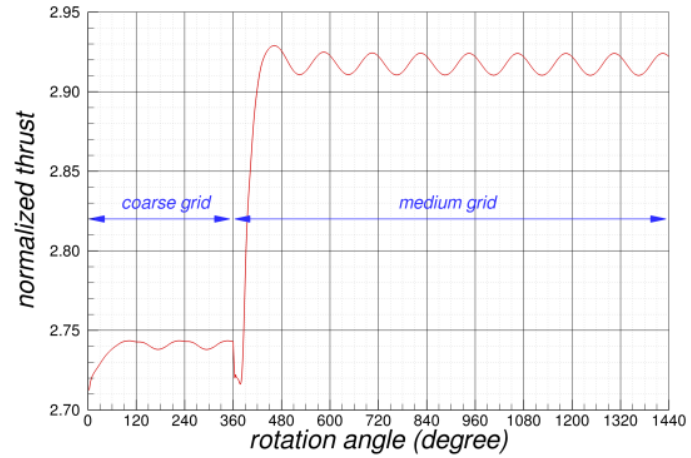

(a) Unsteady flow simulation.

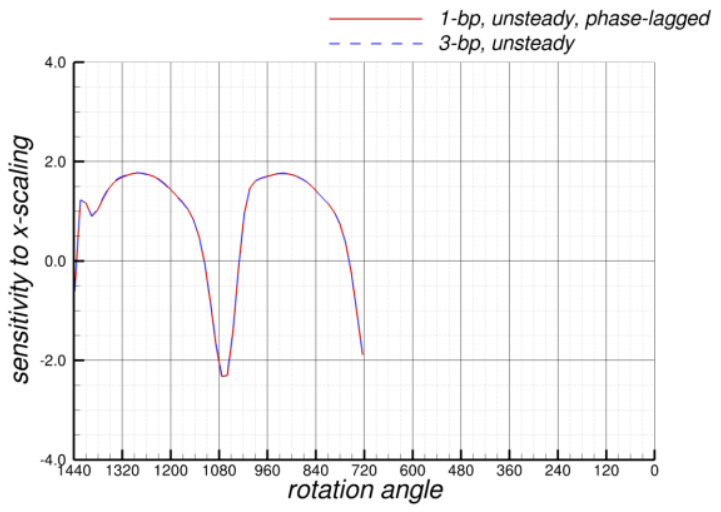

(c) Comparison of unsteady adjoint simulation with 1-BP and 3-BP domains (sensitivity to a single blade).

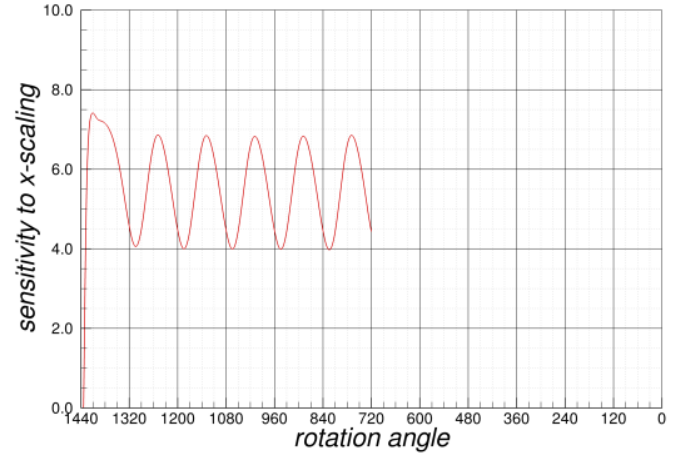

(b) Unsteady adjoint simulation with 3-BP domain (sensitivity to all blades).

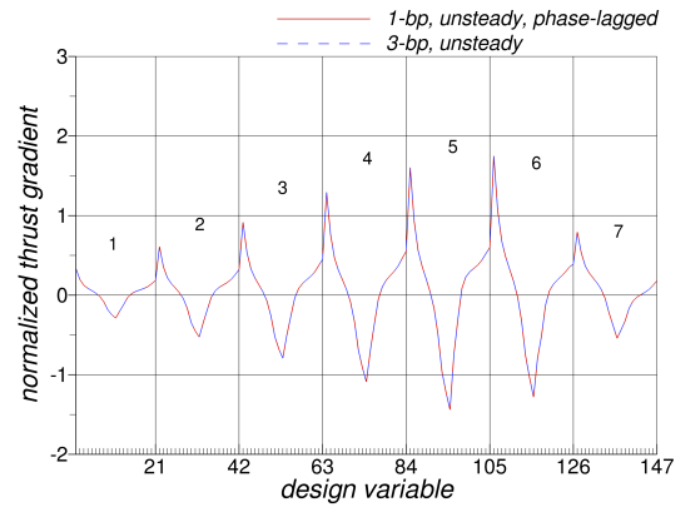

(d) Comparison of the gradients (conventional vs. phase lagged)

Figure 17 Verification of the unsteady adjoint procedure using the phase-lagged boundary condition.

\section{Acknowledgments}

This work has been carried out within the MADELEINE project which has received funding from the European Union's Horizon 2020 research and innovation programme under grant agreement N 769025.

\section{References}

[1] "Flightpath 2050: Europe's Vision for Aviation. Report of the High Level Group on Aviation Research", 2011.

[2] Stokkermans, T., Veldhuis, L., Soemarwoto, B., Fukari, R., Eglin, P., "Breakdown of aerodynamic interactions for the lateral rotors on a compound helicopter", Aerospace Science and Technology, Volume 101, June 2020, 105845, https://doi.org/10.1016/j.ast.2020.105845.

[3] Borer, N.K. and Patterson, M.D., "X-57 High-Lift Propeller Control Schedule Development", AIAA Aviation 2020 Forum, AIAA 2020-3091, June 2020.

[4] Reveles, N.D., Seledic, K., Williams, D. and Blades, E.L., "Rotor/Propeller Model Fidelity Effects on Propeller-Wing Interactional Aerodynamics", AIAA Aviation 2020 Forum, AIAA 2020-2676, June 2020.

[5] Zhou, B.Y., Gauger, N.R., Morelli, M. and Guardone, A., "Simulation and Sensitivity Analysis of a Wing-Tip Mounted Propeller Configuration from the Workshop for Integrated Propeller Prediction (WIPP)", AIAA Aviation 2020 Forum, AIAA 2020-2683, June 2020.

[6] Icke, R. O., Baysal, O., Moy, A., Lopes, L., Zhou, B. Y., and Diskin, B., "Toward Adjoint-Based Aeroacoustic Optimization for Propeller and Rotorcraft Applications," AVIATION 2020 Forum, AIAA 2020-3140, June 2020. 
[7] Wang, L., Diskin, B., Lopes, L.V., Nielsen, E.J., Lee-Rausch, E. and Biedron, R.T., “High-Fidelity AeroAcoustic Optimization Tool for Flexible Rotors", Journal Of The American Helicopter Society 66, 022004 (2021).

[8] Kok, J.C. "A high-order low-dispersion symmetry-preserving finite-volume method for compressible flow on curvilinear grids", Journal of Computational Physics, 228 (2009) 6811-6832.

[9] Ffowcs Williams, J.E. and Hawkings, D.L., "Sound generated by turbulence and surfaces in arbitrary motion", Philosophical Transactions of the Royal Society A 264 (1969) 321-342.

[10] Casalino, D. , "An advanced time approach for acoustic analogy predictions", Journal of Sound and Vibration, 261 (2003) 583-612.

[11] Hascoët,L. and Pascual, L.V., "TAPENADE 2.1 user's guide", https://hal.inria.fr/inria-00069880.

[12] Laban, M. Soemarwoto, B.I., and Kooi, J.W., "Reshaping Engine Nacelles for Testing in Wind Tunnels with Turbofan Propulsion Simulators", AIAA 2005-3703, July 2005.

[13] Soemarwoto, B.I., Boelens, O.J., and Kanakis, A., "Aerodynamic design of gas turbine engine intake duct", Aircraft Engineering and Aerospace Technology, Vol. 88 No. 5, pp. 605-612. https://doi.org/10.1108/AEAT-02-2015-0063.

[14] Soemarwoto, B.I., "Airfoil Optimization using The Navier-Stokes Equations By Means Of The Variational Method", AIAA Paper 98-2401, June 1998.

[15] Soemarwoto, B.I. , Laban, M., Jameson, A., Martins, A.L., and Oskam. B., "Adaptive Aerodynamic Optimization of Regional Jet Aircraft", AIAA Paper 2002-0260, January 2002.

[16] Bez, J.L., Carneiro, A.R., Pavan, P.J., et al., "l/O performance of the Santos Dumont supercomputer. The International Journal of High Performance Computing Applications", The International Journal of High Performance Computing Applications, 2020;34(2):227-245. https://doi.org/10.1177/1094342019868526.

[17] Erdos, J. I., Alzner, E., and McNally, W., "Numerical Solution of Periodic Transonic Flow through a Fan Stage", AlAA Journal 15 (1977) 1559-1568.

[18] Gerolymos, G. A. and Chapin, V., "Generalized Expression of Chorochronic Periodicity in Turbomachinery Blade-Row Interaction", La Recherche Aérospatiale 5 (1991) 69-73.

[19] Strazisar, A. J., Wood, J. R., Hathaway, M. D., and Suder, K. L., "Laser Anemometer Measurements in a Transonic AxialFlow Fan Rotor", NASA Technical Paper 2879, 1989. 